\title{
Consumer attitudes towards personalised nutrition: What do consumers want?
}

\author{
A. Ballantine ${ }^{1}$, B. Stewart-Knox ${ }^{1}$, K. Orr ${ }^{1}$, J. Robinson ${ }^{1}$, M. Duffy ${ }^{1}$, S. Kuznesof ${ }^{2}$ and L. Frewer ${ }^{2}$ \\ ${ }^{1}$ Northern Ireland Centre for Food \& Health (NICHE), University of Ulster, Coleraine, Co. Londonderry BT52 1SA \\ and ${ }^{2}$ Newcastle University, Newcastle upon Tyne, NE1 7RU, UK
}

Personalised nutrition has been defined as a response to differences between individuals and attempts to balance an individual's diet to their specific individual and situational needs, including environmental and psychological factors ${ }^{(1)}$. The Food4Me project has also recently defined personalised nutrition as healthy eating advice that is tailored to suit an individual based on their own personal health status, phenotype and/or genetics ${ }^{(2)}$. The science of nutrigenomics and personalised nutrition is in its infancy and the future success of personalised nutrition and nutrigenomics will depend upon understanding end-user perspectives on new technologies.

The aim of the study was to gain an understanding of consumers' perceptions, preferences and opinions of personalised nutrition among consumers residing in the U.K. and Ireland.

Discussants ( $N$ 68) were recruited from 4 centres (Ulster $n$ 16, Newcastle $n$ 16, Reading $n 20$ and Dublin $n$ 16) through social research agencies. Using a standardised interview schedule developed by the Food4Me partners, discussion was prompted using a number of personalised nutrition scenarios.

Focus group discussions were recorded and transcribed verbatim and thematic content analysis conducted. NVivo9 a qualitative data analysis computer software package was used for storage and retrieval of data.

Although consumers showed some knowledge of personalised nutrition, perceived potential benefits to health, lifestyle and reduction of disease risk, they expressed concerns regarding the delivery of the service, the credibility of the service provider, privacy and the storage and handling of samples. With regard to the service provider, some welcomed the anonymity afforded by internet delivery while others expressed a preference for face-to-face contact. Confidence in ability to conduct self-measurements was also a concern, especially taking a finger prick blood sample. Consumers were also apprehensive about sending personal samples and information via the postal service, inputting information online and the potential for information to be used for discrimination by employers or insurers. The table (below) outlines main themes arising from the discussions.

\begin{tabular}{ll}
\hline Themes & \multicolumn{1}{c}{ Sub-themes } \\
\hline Trust/credibility & $\begin{array}{l}\text { Service provider } \\
\text { Postal service } \\
\text { Self-measurements } \\
\text { Completing questionnaires } \\
\text { Cofficacy }\end{array}$ \\
Perceived risk & $\begin{array}{l}\text { Discrimination by employers or insurers } \\
\text { Reduction in disease risk }\end{array}$ \\
Perceived benefit & Healthier diet or lifestyle \\
\hline
\end{tabular}

These data imply that the on-line delivery of personalised nutrition needs to insure consumers are informed of the credibility and reliability of the service. The service provider should display security policies as well as credentials of those involved. Step-by-step instructions of self-measurements and measures to ensure reliable postal delivery of samples should also be provided to decrease consumers concerns.

This work is supported by the EU funded 7th Framework Food4Me Project.

1. Boland M (2008) Innovat Manag Pol Pract 10, 53-60.

2. Food4me.org 